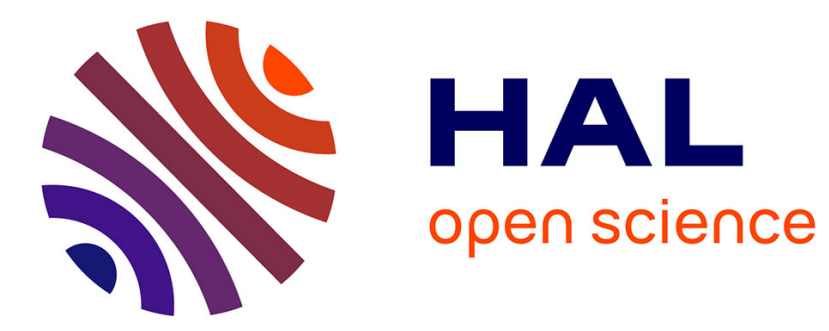

\title{
Spin-Wave Eigen-modes in a Normally Magnetized Nano-pillar
}

\author{
V V Naletov, G. de Loubens, S Borlenghi, Olivier Klein
}

\section{To cite this version:}

V V Naletov, G. de Loubens, S Borlenghi, Olivier Klein. Spin-Wave Eigen-modes in a Normally Magnetized Nano-pillar. Magnonics, pp.3 - 15, 2012, 10.1007/978-3-642-30247-3_1 . cea-01478973

\section{HAL Id: cea-01478973 https://hal-cea.archives-ouvertes.fr/cea-01478973}

Submitted on 28 Feb 2017

HAL is a multi-disciplinary open access archive for the deposit and dissemination of scientific research documents, whether they are published or not. The documents may come from teaching and research institutions in France or abroad, or from public or private research centers.
L'archive ouverte pluridisciplinaire HAL, est destinée au dépôt et à la diffusion de documents scientifiques de niveau recherche, publiés ou non, émanant des établissements d'enseignement et de recherche français ou étrangers, des laboratoires publics ou privés. 


\title{
Chapter 1 \\ Spin-Wave Eigen-modes in a Normally Magnetized Nano-pillar
}

\author{
V.V. Naletov, G. de Loubens, S. Borlenghi, and O. Klein
}

\begin{abstract}
We report on a spectroscopic study of the spin-wave eigen-modes in a circular spin-valve nano-pillar, perpendicularly magnetized along $\hat{z}$. Spectroscopy is performed by Magnetic Resonance Force Microscopy (MRFM). Distinct spectra are measured depending on whether the nano-pillar is excited by a uniform in-plane radio-frequency (RF) magnetic field or by an RF current flowing perpendicularly through the layers. These results are in agreement with micromagnetic simulations of the time decay response of the local magnetization to excitations with different azimuthal symmetries, $(\hat{\boldsymbol{x}}+i \hat{\boldsymbol{y}}) e^{-i \ell \phi}$. This demonstrates that the azimuthal $\ell$-index is the discriminating parameter for the selection rules, as only $\ell=0$ modes are excited by the RF magnetic field, whereas only $\ell=+1$ modes are excited by the RF current, owing to the orthoradial symmetry of the induced RF Oersted field.
\end{abstract}

\subsection{Introduction}

Technological progress in the fabrication of hybrid nanostructures using magnetic metals has allowed the emergence of a new science aimed at utilizing spin dependent effects in the electronic transport properties [1]. An elementary device of spintronics consists of two magnetic layers separated by a normal layer. It exhibits the wellknown giant magneto-resistance (GMR) effect [2,3] and the converse spin transfer effect $[4,5]$.

From an experimental point of view, the precise identification of the spin-wave (SW) eigen-modes in hybrid magnetic nanostructures remains to be done [6-11]. Of particular interest is the exact nature of the modes excited by a polarized current in spin transfer nano-oscillators (STNOs). Here, the identification of the associated symmetry behind each mode is essential. It gives a fundamental insight about their selection rules and about the mutual coupling mechanisms that might exist

V.V. Naletov · G. de Loubens $(\bowtie) \cdot$ S. Borlenghi · O. Klein

Service de Physique de l'État Condensé (URA 2464), CEA Saclay, 91191 Gif-sur-Yvette, France e-mail: gregoire.deloubens@cea.fr

V.V. Naletov

Physics Department, Kazan Federal University, Kazan 420008, Russian Federation 
Fig. 1.1 Schematic representation of the experimental setup. Two independent excitation circuits are used: in red, the circuit allowing the injection of an RF current perpendicular-to-plane through the nano-pillar $\left(i_{\mathrm{rf}}\right.$, red arrow); in blue, the circuit allowing the generation of an $\mathrm{RF}$ in-plane magnetic field $\left(h_{\mathrm{rf}}\right.$, blue arrow $)$

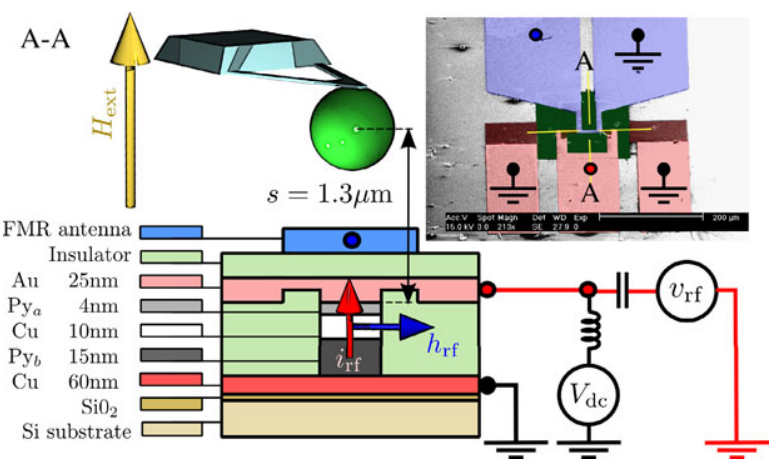

intra or inter STNOs. It also determines the optimum strategy to couple to the autooscillating mode observed when the spin transfer torque compensates the damping, a vital knowledge to achieve phase synchronization in arrays of nano-pillars [12]. These SW modes also have a fundamental influence on the high frequency properties of spin-valve devices, and in particular on the noise of magneto-resistive sensors $[13,14]$.

This work concentrates on a comprehensive identification of the SW eigenmodes in a normally magnetized circular spin-valve nano-pillar, where the axial symmetry is preserved. We shall perform a comparative spectroscopic study of the SW modes excited either by an RF current flowing perpendicularly through the nano-pillar, as used in spin-torque driven ferromagnetic resonance (ST-FMR) $[15,16]$, or by a homogeneous RF in-plane magnetic field, as used in conventional FMR. The experimental spectra are compared to micromagnetic simulations of the time decay response of the local magnetization to a small perturbation of the magnetic configuration. This allows identifying and labeling the observed SW eigenmodes, according to their symmetry and to the dipolar coupling between the two magnetic layers.

The spin-valve structure used in this study is a standard Permalloy $\left(\mathrm{Ni}_{80} \mathrm{Fe}_{20}=\mathrm{Py}\right)$ bi-layer structure sandwiching a $10 \mathrm{~nm}$ copper $(\mathrm{Cu})$ spacer: the thicknesses of the thin $\mathrm{Py}_{a}$ and the thick $\mathrm{Py}_{b}$ layers are respectively $t_{a}=4 \mathrm{~nm}$ and $t_{b}=15 \mathrm{~nm}$. The extended film is patterned by standard e-beam lithography and ionmilling techniques to a nano-pillar of radius $125 \mathrm{~nm}$. The magnetic parameters of this sample have been measured in [17], and are reported in Table 1.1. The top $\mathrm{Cu}$ and bottom Au contact electrodes are shown in red in Fig. 1.1. They are impedance matched to allow for high frequency characterization by injecting an RF current $i_{\mathrm{rf}}$ through the device. Hereafter, spectra associated to SW excitations by this part of the microwave circuit will be displayed in a red tone.

The originality of our design is the addition of an independent top microwave antenna whose purpose is to produce an in-plane RF magnetic field $h_{\mathrm{rf}}$ at the nanopillar location. In Fig. 1.1, this part of the microwave circuit is shown in blue. Injecting a microwave current from a synthesizer inside the top antenna produces a homogeneous in-plane linearly polarized microwave magnetic field, oriented per- 
pendicularly to the stripe direction. Hereafter, spectra associated to SW excitations by this part of the microwave circuit will be displayed in a blue tone.

We shall use in this study a method independent of transport to detect the magnetic resonance inside a spin-valve nanostructure: a Magnetic Resonance Force Microscope (MRFM) [18-20], hereafter named mechanical-FMR. A first decisive advantage of the mechanical-FMR technique is that the detection scheme does not rely on the SW spatial symmetry because it measures the change in the longitudinal component of the magnetization. It thus probes all the excited SW modes, independently of their phase [21,22]. A second decisive advantage is that mechanical-FMR is a very sensitive technique that can measure the magnetization dynamics in nanostructures buried under metallic electrodes [23]. Indeed, the probe is a magnetic particle attached at the end of a soft cantilever and is coupled to the sample through dipolar interaction.

The mechanical detector consists of a $800 \mathrm{~nm}$ diameter sphere of soft amorphous Fe (with $3 \% \mathrm{Si}$ ) glued to the apex of an Olympus Bio-Lever having a spring constant $k \approx 5 \mathrm{mN} / \mathrm{m}$. In our setup, the separation between the center of the spherical probe and the nano-pillar is set to $1.3 \mu \mathrm{m}$ (see Fig. 1.1), which is a large distance considering the lateral size of the sample. The external magnetic field produced by an electromagnet is oriented out-of-plane, exactly along the nano-pillar axis $\hat{z}$. In our study, the strength of the applied magnetic field shall exceed the saturation field $(\approx 8 \mathrm{kOe})$, so that the nano-pillar is studied in the saturated regime. The mechanical sensor measures then the spatial average of the longitudinal component $M_{z}$ of magnetization:

$$
\left\langle M_{z}\right\rangle \equiv \frac{1}{V} \int_{V} M_{z}(\boldsymbol{r}) d^{3} \boldsymbol{r},
$$

where the chevron brackets stand for the spatial average over the volume of the magnetic body. We emphasize that for a bi-layer system, the force signal integrates the contribution of both layers.

The experimental spectra will be shown below in Fig. 1.4(b) and Fig. 1.5(b). The important point is that the two SW spectra excited by $h_{\mathrm{rf}}$ and $i_{\mathrm{rf}}$ are distinct, implying that they have a different origin. It will be shown in the theoretical Sect. 1.2.1 that the RF field and the RF current excitations probe two different azimuthal symmetries $\ell$. Namely, only $\ell=0$ modes are excited by the uniform RF magnetic field, whereas only $\ell=+1$ modes are excited by the orthoradial RF Oersted field associated to the RF current [24]. The mutually exclusive nature of the responses to the uniform and orthoradial symmetry excitations is a property of the preserved axial symmetry, where the azimuthal index $\ell$ is a good quantum number, i.e., different $\ell$-index modes are not mixed and can be excited separately.

\subsection{Identification of the Spin-Wave Modes}

In this section, we discuss the boundary-value problem for SW propagation inside normally magnetized disks, where the confinement leads to a discrete SW spectrum. 
Neglecting the thickness dependence, only three indices are required to label the resonance peaks: the usual azimuthal and radial indices for a single disk $(\ell, m)$, plus an additional index referring to the anti-binding or binding ( $A$ or $B$ ) coupling between the two magnetic layers in mutual dipolar interaction.

\subsubsection{Single Magnetic Disk}

SW eigen-modes are the solutions of the linearized equation of motion of the magnetization, obtained by decomposing the instantaneous magnetization vector $\boldsymbol{M}(t)$ into a static and dynamic component [25]:

$$
\frac{\boldsymbol{M}(t)}{M_{s}}=\hat{\boldsymbol{u}}+\boldsymbol{m}(t)+\mathcal{O}\left(\boldsymbol{m}^{2}\right),
$$

where the transverse component $\boldsymbol{m}(t)$ is the small dimensionless deviation $(|\boldsymbol{m}| \ll 1)$ of the magnetization from the local equilibrium direction, $\hat{\boldsymbol{u}}$. In ferromagnets, $|\boldsymbol{M}|=M_{s}$ is a constant of the motion, so that the local orthogonality condition $\hat{\boldsymbol{u}} \cdot \boldsymbol{m}=0$ is required.

We restrict our study to the case of thin layers so that one can assume that the magnetization dynamics is uniform along the thickness. For a normally magnetized disk, where $\hat{\boldsymbol{u}}=\hat{\boldsymbol{z}}$, the SW modes can be classified according to their behavior under rotations in the $x-y$ plane (2D vector equations with polar coordinates $\rho$ and $\phi$ ):

$$
\boldsymbol{m}_{\ell}=\frac{1}{2}(\hat{\boldsymbol{x}}+i \hat{\boldsymbol{y}}) e^{-i \ell \phi} \psi_{\ell}(\rho)
$$

where the functions $\psi_{\ell}(\rho)$ describe the dependence of the SW mode on the radial coordinate $\rho$ and have to be determined from the dynamical equations of motion. To satisfy the appropriate boundary conditions at the edges of the magnetic body [26], we shall use as the trial vectors the radial profiles of the form $\psi_{\ell}(\rho)=J_{\ell}\left(k_{\ell, n} \rho\right)$, where $J_{\ell}(x)$ is the Bessel function and $k_{\ell, n}$ are $\mathrm{SW}$ wave-numbers determined from the pinning conditions at the disk boundary $\rho=R$. For our experimental conditions $\left(t_{a}, t_{b} \ll R\right)$, the pinning is almost complete, and we shall use $k_{\ell, n}=\kappa_{\ell, n} / R$, where $\kappa_{\ell, n}$ is the $n$th root of the Bessel function of the $\ell$ th order. The set of Bessel function form here a complete basis in the space of vector functions $\boldsymbol{m}$.

Figure 1.2 shows a color representation of the Bessel spatial patterns for different values of the index $v=\ell, n$. We restrict the number of panels to two values of the azimuthal mode index, $\ell=0,+1$, with the radial index varying between $n=$ $0,1,2$. In our color code, the hue indicates the phase (or direction) of the transverse component $\boldsymbol{m}_{v}$, while the brightness indicates the amplitude of $\left|\boldsymbol{m}_{v}\right|^{2}$. The nodal positions are marked in white. A node is a location where the transverse component vanishes, i.e., the magnetization vector is aligned along the equilibrium axis. This coding scheme provides a distinct visualization of the phase and amplitude of the precession profiles. The black arrows are a snapshot of the $\boldsymbol{m}_{v}$ vectors in the disk and are all rotating synchronously in-plane at the SW eigen-frequency. 
Fig. 1.2 Color representation of the Bessel spatial patterns for different values of the azimuthal mode index $\ell$ (by row) and radial mode index $n$ (by column). The arrows are a snapshot of the transverse magnetization $\boldsymbol{m}_{v}$, labeled by the index $v=\ell, n$
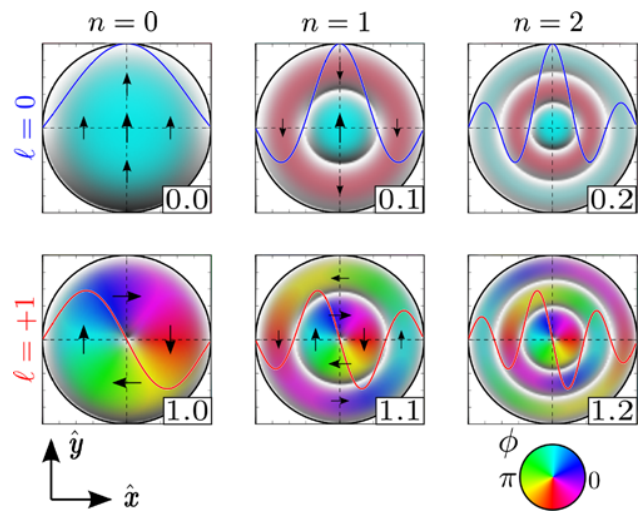

The top left panel shows the $v=0,0(\ell=0, n=0)$ mode, also called the uniform mode. It usually corresponds to the lowest energy mode since all the vectors are pointing in the same direction at all time. Below is the $\ell=+1, n=0$ mode. It corresponds to SWs that are rotating around the disk in the same direction as the Larmor precession. The corresponding phase is in quadrature between two orthogonal positions and this mode has a node at the center of the disk. The variation upon the $n=0,1,2$ index ( $\ell$ being fixed) shows higher order modes with an increasing number of nodal rings. Each ring separates regions of opposite phase along the radial direction. All these spatial patterns preserve the rotation invariance symmetry.

The coupling to an external coherent source depends primarily on the $\ell$-index [27] as the excitation efficiency is proportional to the overlap integral

$$
h_{v} \propto\left\langle\overline{\boldsymbol{m}}_{v} \cdot \boldsymbol{h}_{1}\right\rangle
$$

where $\boldsymbol{h}_{1}(\boldsymbol{r})$ is the spatial profile of the external excitation field. It can be easily shown that a uniform RF magnetic field, $\boldsymbol{h}_{1}=h_{\mathrm{rf}} \boldsymbol{x}$, can only excite $\ell=0 \mathrm{SW}$ modes. Obviously, the largest overlap is obtained with the so-called uniform mode $(n=0)$. Higher radial index modes $(n \neq 0)$ still couple to the uniform excitation but with a strength that decreases as $n$ increases $[19,28]$. The $\ell \neq 0$ normal modes, however, are hidden because they have strictly no overlap with the excitation. In contrast, the RF current-created Oersted field, $\boldsymbol{h}_{1}=h_{\mathrm{Oe}}(\rho)(-\sin \phi \hat{\boldsymbol{x}}+\cos \phi \hat{\boldsymbol{y}})$ has an orthoradial symmetry and can only excite $\ell=+1 \mathrm{SW}$ modes.

\subsubsection{Double Magnetic Disks}

The interaction between two identical magnetic layers leads to the splitting of the uniform mode in each layer into two collective modes: the binding and anti-binding modes. This has been observed in interlayer-exchange-coupled thin films [29] and in trilayered wires where the two magnetic stripes are dipolarly coupled [30]. In the 
case where the two magnetic layers are not identical (different geometry or magnetic parameters), this general picture continues to subsist. Although both isolated layers have eigen-modes with different eigen-frequencies, the collective magnetization dynamics in each layer are still symmetrically or anti-symmetrically coupled. But here, the precession of magnetization can be more intense in one of the two layers and the spectral shift of the coupled SW modes with respect to the isolated SW modes is reduced, as it was observed in both the dipolarly- [30] and exchangecoupled cases [31].

Here, we assume that the dominant coupling mechanism between the two magnetic disks in the nano-pillar is the dipolar interaction. We neglect any exchange coupling between the magnetic layers mediated through the normal spacer or any coupling associated to pure spin currents $[7,32]$ in our all-metallic spin-valve structure. Our nano-pillar consists of two dislike magnetic layers, having different thicknesses, with $t_{a}<t_{b}$, and different saturation magnetizations, with $M_{a}<M_{b}$. In the normally saturated state which is considered here, this difference of magnetization leads to a difference in the internal demagnetizing field of each layer (self dipolar coupling) and in the stray field of one layer on the other one (cross dipolar coupling). As a result, the eigen-frequency of the uniform mode of the thin layer is larger than the one of the thick layer, $\omega_{a}>\omega_{b}$. This is represented in Fig. 1.3(a), on both sides of the bi-layer diagram, where we have reported on an energy scale their relative positions.

In the perpendicular geometry, the strength of the dynamical dipolar coupling $\Omega$ between the layers depends on the cross tensor element $j j^{\prime}$, associated to the in-plane component of the dipolar magnetic field produced inside layer $j$ by the in-plane component of the magnetization of layer $j^{\prime}$ (see [17, 33]). It is attractive (lower in energy) when both layers precess in anti-phase because the dynamical dipolar charges in each layer are alternate (anti-ferromagnetic coupling). Thus the binding state corresponds to a collective motion where the two layers vibrate antisymmetrically $(B)$ and the anti-binding state to a collective motion where they vibrate symmetrically $(A)$. In this case, the larger of the frequencies $\left(\omega_{a}\right)$ shifts up by

$$
\Delta \omega=\frac{\Omega^{2}}{\omega_{a}-\omega_{b}},
$$

while the smaller one $\left(\omega_{b}\right)$ shifts down by the same amount. This effect is summarized in Fig. 1.3.

A numerical estimate of the coupling strengths between the lowest energy SW modes in each disk can be found in [17]. For the experimental parameters, $\Omega / 2 \pi \simeq$ $0.5 \mathrm{GHz}$. This coupling is almost an order of magnitude smaller than the frequency splitting $\omega_{a}-\omega_{b}$, caused mainly by the difference of effective magnetizations of two disks: $\gamma 4 \pi\left(M_{b}-M_{a}\right) \simeq 2 \pi \cdot 4.5 \mathrm{GHz}$. As a result, the shift of the resonance frequencies due to the dipolar coupling is negligible, $\Delta \omega / 2 \pi \simeq 0.06 \mathrm{GHz}$. The effect of dynamic dipolar interaction is more pronounced for the level of mode hybridization. For instance, at the frequency $\omega_{A} \approx \omega_{a}$, the ratio between the precession 


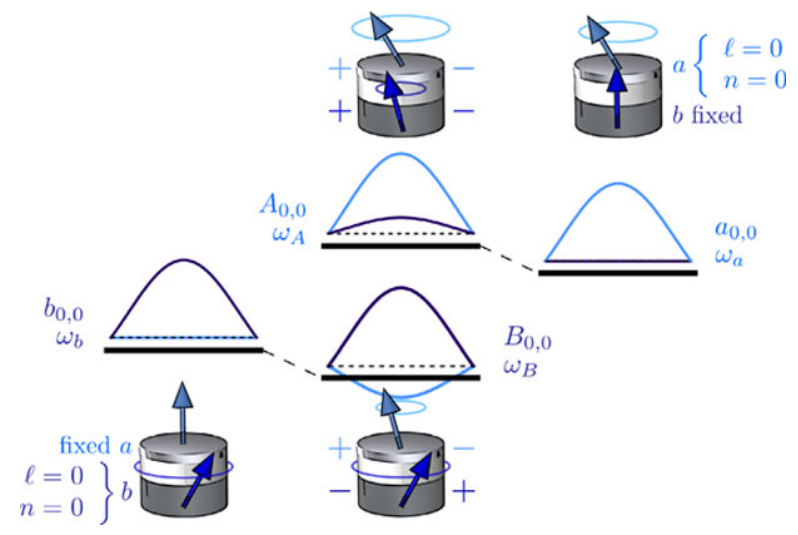

Fig. 1.3 Schematic representation of the coupled dynamics between two different magnetic disks. When the two disks are dynamically coupled through the dipolar interaction, the binding state $B$ corresponds to the two layers oscillating in anti-phase at $\omega_{B}$, with the precession occurring mostly in the thick layer, whereas the anti-binding state $A$ corresponds to the layers oscillating in phase at $\omega_{A}$, with the precession mostly in the thin layer

amplitudes in the two layers is given by

$$
\left|c_{b} / c_{a}\right|_{\omega_{A}}=\Delta \omega /\left(\gamma h_{a, b}\right) \simeq \frac{\Omega}{\omega_{a}-\omega_{b}} .
$$

For the experimental parameters, $\Omega /\left(\omega_{a}-\omega_{b}\right) \approx 0.1$, i.e., the precession amplitude in the disk $b$ is about $10 \%$ of that in the disk $a$. Thus, although the dipolar coupling induces a small spectral shift (second order in the coupling parameter (1.5)), its influence in the relative precession amplitude is significant (first order in the coupling parameter (1.6)).

\subsubsection{Micromagnetic Simulations vs. Mechanical-FMR Experiments}

Although the analytical formalism presented above allows estimating the spectrum, several approximations have been made. In particular, we have assumed total pinning at the disks boundary for the SW modes and no variation of the precession profile along the disks thicknesses (2D model), and we have neglected the dependence on the mode index $v$ of the dynamic dipolar coupling. Instead of developing a more complex analytical formalism, we have performed a complete calculation of the SW spectra inside our nano-pillar sample using the open source micromagnetic simulation package Nmag [34]. Nmag is a finite element solver based on the general purpose multi-physics library Nsim. It is developed by the group of H. Fangohr and T. Fischbacher in the School of Engineering Sciences at the University of Southampton. 
Table 1.1 Magnetic parameters of the thin $\mathrm{Py}_{a}$ and thick $\mathrm{Py}_{b}$ layers measured by mechanicalFMR in the nano-pillar

\begin{tabular}{lllll}
\hline $4 \pi M_{a}(\mathrm{G})$ & $\alpha_{a}$ & $4 \pi M_{b}(\mathrm{G})$ & $\alpha_{b}$ & $\gamma\left(\mathrm{rad} \mathrm{s}^{-1} \mathrm{G}^{-1}\right)$ \\
\hline $8.0 \times 10^{3}$ & $1.4 \times 10^{-2}$ & $9.6 \times 10^{3}$ & $0.85 \times 10^{-2}$ & $1.87 \times 10^{7}$ \\
\hline
\end{tabular}

In this simulation, the full three-dimensional (3D) dynamics of the bi-layer system is calculated. The thin layer is discretized with a mesh of $4 \mathrm{~nm}$ (equal to its thickness), and the thick layer with a mesh of $3 \mathrm{~nm}$. The numbers of nodes are respectively 6135 in the thin layer and 37598 in the thick layer. The magnetic parameters introduced in the code are the ones reported in Table 1.1. The magnetization vector is assumed to be uniform inside each cell, which is valid because the cell size is smaller than the exchange length $\Lambda \simeq 5 \mathrm{~nm}$ in Permalloy. We emphasize that the simulations incorporate the perturbing presence of the magnetic sphere attached on the MRFM cantilever. Moreover, the $10 \mathrm{~nm}$ thick $\mathrm{Cu}$ spacer is replaced by vacuum, so that the layers are only coupled trough the dipolar interaction (spin diffusion effects are absent).

The first step is to calculate the equilibrium configuration of the normally magnetized nano-pillar at $H_{\text {fix }}=10 \mathrm{kOe}$. The external magnetic field is applied exactly along $\hat{z}$ and the spherical probe with a magnetic moment $m=2 \times 10^{-10} \mathrm{emu}$ is placed on the axial symmetry axis at a distance $s=1.3 \mu \mathrm{m}$ above the upper surface of the nano-pillar (see Fig. 1.1). The convergence criterion introduced in the code is $1 / M_{S} d \boldsymbol{M} / d t<1 \% \mathrm{~ns}$. The result reveals that the equilibrium configuration is almost uniformly saturated along $\hat{z}$. Still, a small radial flaring $\left(<5^{\circ}\right)$ of the magnetization from $\hat{z}$ is observed at the periphery of the thick and thin layers.

In order to calculate the SW spectrum corresponding to a given excitation of this equilibrium state at $H_{\mathrm{fix}}=10 \mathrm{kOe}$, we record the time decay response of the local magnetization to a small perturbation of the magnetic configuration, and we Fourier transform the ringdown of the transverse magnetization [35]. Two different initial conditions are simulated. For the conventional FMR spectrum, in which a uniform $\mathrm{RF}$ field is used to excite SW modes, we use the following perturbation vector field:

$$
\boldsymbol{m}=\vartheta \sum_{n<6} J_{0}\left(k_{0 n} \rho\right) \hat{\boldsymbol{x}} .
$$

This form corresponds to an excitation which puts the same energy in the lowest $6 n$-index modes with azimuthal index $\ell=0$. For the spectrum excited by an RF current flowing through the nano-pillar, which creates an orthoradial RF Oersted field in the magnetic volume, we use:

$$
\boldsymbol{m}=\vartheta \sum_{n<6} J_{1}\left(k_{1 n} \rho\right)(-\sin \phi \hat{\boldsymbol{x}}+\cos \phi \hat{\boldsymbol{y}}) .
$$

At time $t=0$, we add at every mesh the perturbation vector field defined in (1.7) or in (1.8) to the local unit vector along the equilibrium magnetization. The first one 

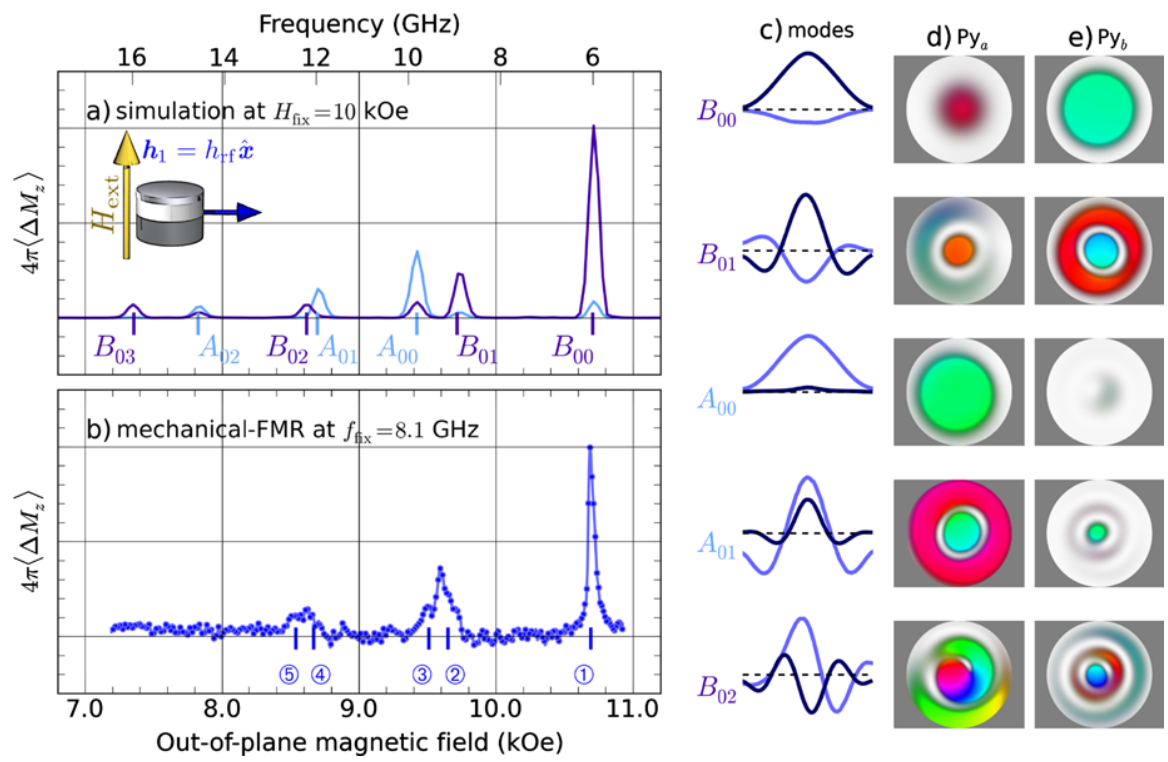

Fig. 1.4 Panel (a) is the numerically calculated spectral response to a uniform excitation field $\boldsymbol{h}_{1} \propto \hat{\boldsymbol{x}}$, from a 3D micromagnetic simulation performed at $H_{\mathrm{fix}}=10 \mathrm{kOe}$. The peaks are labeled according to their precession profiles shown in (c), (d), and (e). A light (dark) color is used to indicate the energy stored in the thin (thick) layer. Panel (b) shows the experimental spectrum measured by mechanical-FMR when exciting the nano-pillar by a homogeneous RF magnetic field at $f_{\mathrm{fix}}=8.1 \mathrm{GHz}$

corresponds to a uniform tilt (i.e., in the same azimuthal direction) of the magnetic moments located in the center region of the two disks. The second one corresponds to an orthoradial tilt of the magnetization at the periphery of the nano-pillar. The perturbation angle $\vartheta=0.01 \ll 1$ leads to a deviation from the local equilibrium axis of less than $1^{\circ}$ degree, which is small enough to ensure that non-linear effects are weak in the simulated dynamics. We then compute the magnetization decay inside the whole sample. The ringdown of the transverse magnetization is recorded in a $10 \mathrm{~ns}$ time window with a sampling interval of $5 \mathrm{ps}$.

At every time step, a spatial average of the in-plane component of the magnetization is recorded. In the case of the initial condition of (1.7), we construct the complex reduced magnetization $\langle\boldsymbol{m} \cdot \hat{\boldsymbol{x}}\rangle$ inside each layer $j=a, b$. In the case of the initial condition of (1.8), a different spatial average is used, $\left\langle\boldsymbol{m} \cdot(\hat{\boldsymbol{x}}+i \hat{\boldsymbol{y}}) e^{-i \phi}\right\rangle$, where $\phi$ is the local azimuthal coordinate of the mesh node. Then, the power density spectrum of the time decay of the full averaged reduced magnetization is calculated in each layer using a complex Fourier transform.

The comparison between the simulated and experimental spectra corresponding to a uniform RF field excitation and to an RF current flowing through the nano-pillar is presented in Figs. 1.4(a)-(b) and 1.5(a)-(b), respectively. The frequency scale of the simulation is in correspondence with the field-sweep scale of the experiments 

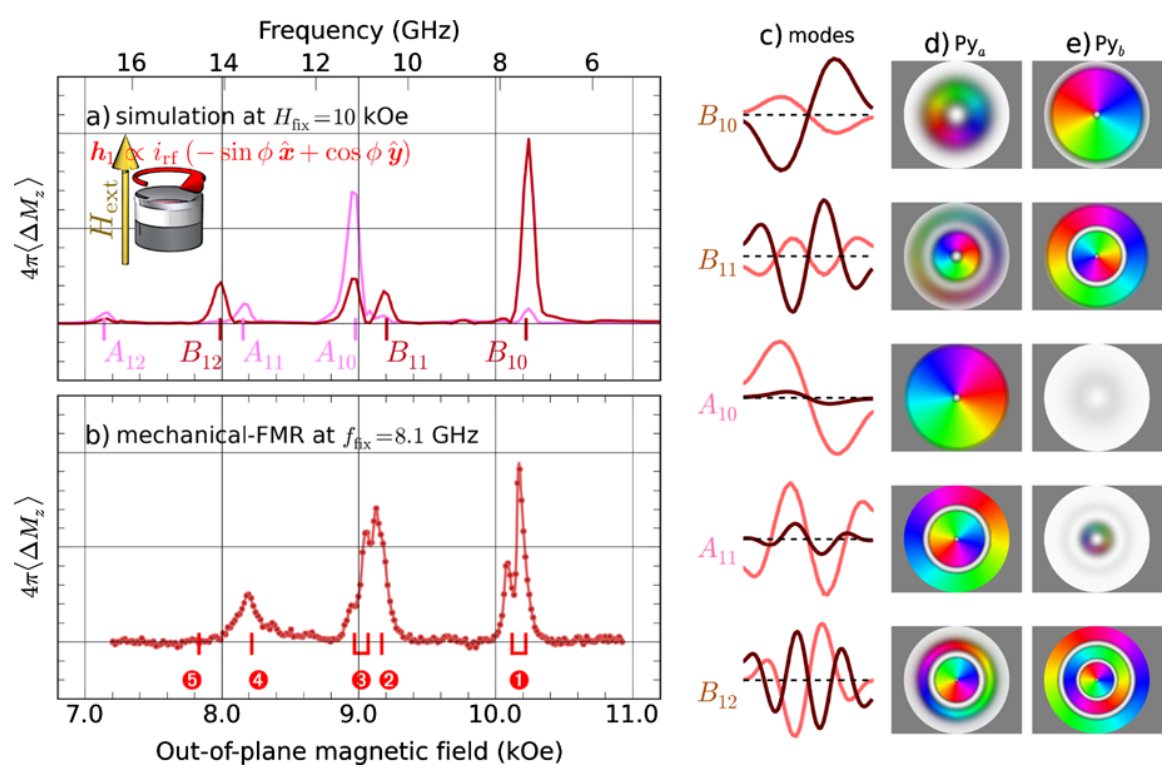

Fig. 1.5 Panel (a) is the simulated spectral response to an orthoradial excitation field $\boldsymbol{h}_{1} \propto-\sin \phi \hat{\boldsymbol{x}}+\cos \phi \hat{\boldsymbol{y}}$. Panel (b) is the experimental spectrum measured by mechanical-FMR for an RF current excitation. Panels (c)-(e) show the simulated precession patterns of the eigen-vectors

performed at fixed RF frequency $f_{\text {fix }}=8.1 \mathrm{GHz}$ through the affine transformation $H_{\text {ext }}-H_{\text {fix }}=2 \pi\left(f-f_{\text {fix }}\right) / \gamma$, as seen from the frequency and field scales above Fig. 1.4(a) and below Fig. 1.4(b), respectively. Since in the simulations, we have access to the dynamics inside each layer, a light (dark) color is used to indicate the power spectrum of the thin (thick) layer. The resonance peaks are labeled according to the modes precession profiles, which are visualized by calculating the Fourier transform of the magnetization decay at every mesh node. The results are shown in the right side of Figs. 1.4 and 1.5, along with the precession profiles along the median direction. The $2 \mathrm{D}$ views of the spatial distribution of the transverse magnetization in the thin $\mathrm{Py}_{a}$ and thick $\mathrm{Py}_{b}$ layers are shown using the same color code as in Fig. 1.2.

Let us first focus on Fig. 1.4 which enables identifying the SW modes excited by a uniform RF magnetic field. The five peaks labeled on the simulated spectrum are in correspondence with the experimental resonance peaks. From the color code panels of Fig. 1.4(d)-(e), we see that these five modes have the same phase along the azimuthal direction, which is the character of the $\ell=0$ index. The largest peak in the simulation occurs at the same field as the experimental peak at $H_{(1)}$. This lowest energy mode corresponds to the most uniform mode with the largest wave-vector and no node along the radial direction, thus it has the index $n=0$. For this mode, the thick layer is mainly precessing, with the thin layer oscillating in anti-phase, as can be seen from the representations of its spatial profile in Fig. 1.4(c), so it bears the 
binding index $B$. Therefore, it should be labeled $B_{00}$, which confirms the character of the lowest energy collective mode in the perpendicular geometry discussed in Sect. 1.2.2. We also note that the relative amplitude of the precession in the two layers found in the simulation is roughly in agreement with the predicted value from the analytical model, see (1.6). The same analysis can be made for the second peak, labeled $B_{01}$, which occurs close to the experimental peak at $H_{2}$. It also corresponds to a resonance mainly in the thick layer, and its color representation shows that this is the first radial harmonic $(n=1)$, with one line of node in the radial direction. Again, the thin layer is oscillating in anti-phase, with the same radial index $n=1$, as clearly shown by the mode profile along the median direction. The third peak is labeled $A_{00}$ and is located close to the experimental peak at $H_{3}$. It corresponds this time to a uniform $(n=0)$ precession mainly located in the thin layer, in agreement with the experimental analysis presented in [17]. In this mode, the thick layer is also slightly vibrating in phase with the thin layer (anti-binding index $A$ ). The other two modes, $A_{01}$ and $B_{02}$, correspond to higher radial harmonics of $\ell=0$ modes in the nanopillar, at $H_{(}$and $H_{(5}$. Due to the proximity between these two modes, the precession profile of the mode $B_{02}$ appears somewhat hybridized with the precession profile of the mode $A_{01}$. Finally, one can also check from the simulations the independence of the precession profiles on the thickness (within the uncertainty of the calculated profile, which is about $\pm 2 \%$ ). This confirms the validity of the $2 \mathrm{D}$ approximation and explains the performance of the analytical model [17].

Let us now turn to Fig. 1.5(b), corresponding to the spectroscopic response to an RF current of same frequency $8.1 \mathrm{GHz}$ flowing perpendicularly through the nanopillar. The SW spectrum is acquired under the exact same conditions as for standard FMR, i.e., the spherical magnetic probe of the mechanical-FMR detection is kept at the same location above the sample. The striking result is that the position of the peaks in Figs. 1.4(b) and 1.5(b) do not coincide. More precisely, there seems to be a translational correspondence between the two spectra, which are shifted in field by about $0.5 \mathrm{kOe}$ from each other. The comparison with Fig. 1.5(a) enables us to identify the SW modes excited by the orthoradial RF Oersted field produced by the RF current. Here again, the peaks of the simulated power spectrum in Fig. 1.5(a) are in good correspondence with the experimental resonance peaks in Fig. 1.5(b). Repeating the same analysis as above, the peaks follow the same sequence of $A / B$, $n$ indexation and only differ by their $\ell$-index. This explains the translational correspondence between the SW spectra of Figs. 1.4(b) and 1.5(b). Finally, we point out that the dipolar pinning at the boundaries of each disk in the modes displayed in Fig. 1.5(c) is not trivial [36]. The general trend observed here is that the thin layer is less pinned than the thick layer for symmetric modes, and vice versa.

Thus, these full 3D micromagnetic simulations allow the identification of the SW modes probed experimentally by both a uniform RF magnetic field and an RF current flowing through the nano-pillar, i.e., of their respective selection rules. They also give a deeper insight on the collective nature of the magnetization dynamics in the nano-pillar discussed in Sect. 1.2.2. 


\subsection{Conclusion}

In summary, we used the MRFM technique [20] to study the SW eigen-modes in the prototype of a STNO - a normally magnetized nano-pillar composed of two magnetic layers in dipolar interaction. We were able to compare the SW spectra of this passive STNO excited by a uniform in-plane RF magnetic field and by an RF current flowing perpendicularly through the layers. We found that distinctly different SW modes (having azimuthal indices $\ell=0$ and $\ell=+1$, respectively) are excited by the two above mentioned excitation methods. We also developed a simple analytic theory providing a comprehensive labeling of all the SW eigen-modes of a magnetic nano-pillar in the studied axially symmetric case. This labeling requires three independent indices: the usual azimuthal and radial indices $\ell$ and $n$ used for the SW modes of a single magnetic disk, and an additional index referring to the binding or anti-binding ( $B$ or $A$ ) dipolar coupling between the two magnetic disks.

The obtained experimental and analytic results were also compared to the results of 3D micromagnetic simulations obtained with the Nmag package. We learned that the $\ell$-index, related to the azimuthal symmetry of the SW modes, is the discriminating parameter for the selection rules of the SW mode excitation. We believe that our results are important for the optimization of the characteristics of nano-spintronic devices, and in particular STNOs, and for the experimental determination of the STNO parameters.

Acknowledgements We thank V. Cros, J. Grollier, H. Hurdequint, C. Ulysse, G. Faini, V.S. Tiberkevich, and A.N. Slavin for their help and support. This research was partially supported by the European Grant Master (NMP-FP7 212257) and by the French Grant Voice (ANR-09NANO-006-01).

\section{References}

1. S. Wolf, D.D. Awschalom, R.A. Buhrman, J. Daughton, S. von Molnar, M.L. Roukes, A.Y. Chtchelkanova, D.M. Treger, Science 294, 1488 (2001)

2. M.N. Baibich, J.M. Broto, A. Fert, F.N.V. Dau, F. Petroff, P. Etienne, G. Creuzet, A. Friederich, J. Chazelas, Phys. Rev. Lett. 61(21), 2472 (1988)

3. G. Binasch, P. Grunberg, F. Saurenbach, W. Zinn, Phys. Rev. B 39(7), 4828 (1989)

4. J. Slonczewski, J. Magn. Magn. Mater. 159, L1 (1996). doi:10.1016/0304-8853(96)00062-5

5. L. Berger, Phys. Rev. B 54(13), 9353 (1996)

6. V.E. Demidov, S.O. Demokritov, B. Hillebrands, M. Laufenberg, P.P. Freitas, Appl. Phys. Lett. 85(14), 2866 (2004). doi:10.1063/1.1803621

7. G. Woltersdorf, O. Mosendz, B. Heinrich, C.H. Back, Phys. Rev. Lett. 99(24), 246603 (2007). doi:10.1103/PhysRevLett.99.246603

8. G. de Loubens, V.V. Naletov, O. Klein, J. Ben Youssef, F. Boust, N. Vukadinovic, Phys. Rev. Lett. 98(12), 127601 (2007)

9. G. de Loubens, V.V. Naletov, M. Viret, O. Klein, H. Hurdequint, J. Ben Youssef, F. Boust, N. Vukadinovic, J. Appl. Phys. 101, 09F514 (2007)

10. G. Gubbiotti, M. Madami, S. Tacchi, G. Carlotti, H. Tanigawa, T. Ono, J. Phys. D, Appl. Phys. 41(13), 134023 (2008). doi:10.1088/0022-3727/41/13/134023

11. P.S. Keatley, V.V. Kruglyak, A. Neudert, E.A. Galaktionov, R.J. Hicken, J.R. Childress, J.A. Katine, Phys. Rev. B 78(21), 214412 (2008). doi:10.1103/PhysRevB.78.214412 
12. A. Slavin, V. Tiberkevich, IEEE Trans. Magn. 45(4), 1875 (2009). doi:10.1109/ TMAG.2008.2009935

13. A.V. Nazarov, H.S. Cho, J. Nowak, S. Stokes, N. Tabat, Appl. Phys. Lett. 81(24), 4559 (2002). doi:10.1063/1.1521578

14. N. Stutzke, S.L. Burkett, S.E. Russek, Appl. Phys. Lett. 82, 91 (2003). doi:10.1063/1.1534386

15. J.C. Sankey, P.M. Braganca, A.G.F. Garcia, I.N. Krivorotov, R.A. Buhrman, D.C. Ralph, Phys. Rev. Lett. 96(22), 227601 (2006)

16. W. Chen, J.M.L. Beaujour, G. de Loubens, A.D. Kent, J.Z. Sun, Appl. Phys. Lett. 92(1), 012507 (2008). doi:10.1063/1.2827570

17. V.V. Naletov, G. de Loubens, G. Albuquerque, S. Borlenghi, V. Cros, G. Faini, J. Grollier, H. Hurdequint, N. Locatelli, B. Pigeau, A.N. Slavin, V.S. Tiberkevich, C. Ulysse, T. Valet, O. Klein, Phys. Rev. B 84, 224423 (2011). doi:10.1103/PhysRevB.84.224423

18. Z. Zhang, P.C. Hammel, P.E. Wigen, Appl. Phys. Lett. 68, 2005 (1996)

19. V. Charbois, V.V. Naletov, J. Ben Youssef, O. Klein, J. Appl. Phys. 91, 7337 (2002)

20. O. Klein, G. de Loubens, V.V. Naletov, F. Boust, T. Guillet, H. Hurdequint, A. Leksikov, A.N. Slavin, V.S. Tiberkevich, N. Vukadinovic, Phys. Rev. B 78(14), 144410 (2008). doi:10.1103/PhysRevB.78.144410

21. G. de Loubens, V.V. Naletov, O. Klein, Phys. Rev. B 71(18), 180411 (2005)

22. V.V. Naletov, G. de Loubens, V. Charbois, O. Klein, V.S. Tiberkevich, A.N. Slavin, Phys. Rev. B 75(14), 140405 (2007)

23. G. de Loubens, A. Riegler, B. Pigeau, F. Lochner, F. Boust, K.Y. Guslienko, H. Hurdequint, L.W. Molenkamp, G. Schmidt, A.N. Slavin, V.S. Tiberkevich, N. Vukadinovic, O. Klein, Phys. Rev. Lett. 102(17), 177602 (2009). doi:10.1103/PhysRevLett.102.177602

24. R.E. Arias, D.L. Mills, Phys. Rev. B 79, 144404 (2009)

25. A.G. Gurevich, G.A. Melkov, Magnetization Oscillations and Waves (CRC Press, Boca Raton, 1996)

26. K.Y. Guslienko, S.O. Demokritov, B. Hillebrands, A.N. Slavin, Phys. Rev. B 66, 132402 (2002)

27. J.F. Dillon, J. Appl. Phys. 31(9), 1605 (1960)

28. R.W. Damon, J.R. Eshbach, J. Phys. Chem. Solids 19, 308 (1961)

29. M. Belmeguenai, T. Martin, G. Woltersdorf, M. Maier, G. Bayreuther, Phys. Rev. B 76(10), 104414 (2007). doi:10.1103/PhysRevB.76.104414

30. G. Gubbiotti, M. Kostylev, N. Sergeeva, M. Conti, G. Carlotti, T. Ono, A.N. Slavin, A. Stashkevich, Phys. Rev. B 70(22), 224422 (2004). doi:10.1103/PhysRevB.70.224422

31. J. Ben Youssef, A. Layadi, J. Appl. Phys. 108(5), 053913 (2010). doi:10.1063/1.3476269

32. B. Kardasz, O. Mosendz, B. Heinrich, Z. Liu, M. Freeman, J. Appl. Phys. 103(7), 07C509 (2008). doi:10.1063/1.2834399

33. O. Dmytriiev, T. Meitzler, E. Bankowski, A. Slavin, V. Tiberkevich, J. Phys. Condens. Matter 22, 136001 (2010)

34. T. Fischbacher, M. Franchin, G. Bordignon, H. Fangohr, IEEE Trans. Magn. 43(6), 2896 (2007)

35. R.D. McMichael, M.D. Stiles, J. Appl. Phys. 97(10), 10J901 (2005)

36. M.P. Kostylev, A.A. Stashkevich, N.A. Sergeeva, Y. Roussigné, J. Magn. Magn. Mater. 278, 397 (2004). doi:10.1016/j.jmmm.2003.11.400 